\title{
SISTEMA DO TRÁFEGO INTERNACIONAL: SUBSÍDIO PARA AS POLÍTICAS PÚBLICAS
}

\author{
SISTEMA DE TRÁFICO INTERNACIONAL: SUBSÍDIO A LAS POLÍTICAS PÚBLICAS
}

INTERNATIONAL TRAFFIC SYSTEM: SUBSIDY FOR PUBLIC POLICIES

\author{
José César Pontes Moreira ${ }^{1}$ \\ Paulo Roberto de Lima Carvalho ${ }^{2}$ \\ Cláudio Rogério Carneiro Pimentel ${ }^{3}$
}

RESUMO: O objetivo deste trabalho é analisar o Sistema do Tráfego Internacional (STI) de forma a identificar os elementos existentes na base de dados passíveis de tratamento estatístico, aptos a subsidiar os governos Federal, Estadual e Municipal na elaboração de políticas públicas nos diversos setores. Para isso, será utilizada a base de dados "online" do Sistema do Tráfego Internacional (STI), do DPF, a amostra será constituída de dados de cinco anos, compreendido no período de or janeiro de 2008 a 31 de Dezembro de 2012, em nível nacional. Os métodos observacional, entrevista informal, da pesquisa bibliográfica, da pesquisa documental e aplicação de questionário. A coleta de dados primários foi realizada mediante acesso direto ao STI. As entrevistas foram realizadas com is especialistas, servidores públicos das esferas federal, estadual e municipal, das áreas da segurança, da educação e saúde. Os especialistas apontaram categorias como útil para o controle do fluxo migratório, além de servir para quantificar e dimensionar os serviços de migração para adequação dos padrões internacionais dado foi apontado como útil para o controle do fluxo migratório, além de servir para quantificar e dimensionar os serviços de migração para adequação dos padrões internacionais. Concluiu-se que há falta de integração das bases de dados dos sistemas informáticos públicos, o que contribui para um aumento dos custos de produção de informação, em razão de dificultar o reaproveitamento de dados armazenados por outros órgãos da administração pública, sendo viável a aplicação prática dos respectivos dados atualmente disponíveis.

PALAVRAS-CHAVE: Gestão pública. Tráfego internacional. Segurança. Saúde. Economia.

\footnotetext{
1 Doutorando em Educação - Programa de Pós-Graduação em Educação da Universidade Federal do Ceará (UFC), mestrado em Economia Rural e graduado em Ciências Econômicas pela UFC. Atualmente é Diretor de Extensão e economista da UFC. Tem experiência na área de Economia, com ênfase em Economia Institucional, atuando principalmente nos seguintes temas: administração pública, agricultura familiar, gestão pública, serviço público e cooperativismo.E-mail: cesarpontes@ufc.br.
}

${ }^{2}$ Advogado e administrador de empresas, professor, palestrante, escritor e articulista. Mestre em Planejamento e Políticas Públicas pela Universidade Estadual do Ceará. Especialista em Execução de Políticas de Segurança Pública; especialista em Direito Processual Civil pela Universidade Gama Filho (2009). Integrante do Laboratório de Políticas de Defesa e Segurança Pública (LEPDESP). E-mail: contato@paulocarvalho.adv.br.

3 Teólogo formado pela Faculdade de Teologia de Fortaleza - (FATEFOR) em 2015. Graduando em Tecnologia em Gestão da Qualidade pela Universidade Federal do Ceará - (UFC). Técnico-administrativo em educação na Universidade federal do Ceará. Chefe da Seção de Apoio à Educação Continuada da Pró-reitora de Extensão da UFC. E-mail: rogerufc@gmail.com. 
RESUMEN: El objetivo de este trabajo es analizar el Sistema de Tráfico Internacional (STI) con el fin de identificar los elementos existentes en la base de datos sujetos a tratamiento estadístico, capaces de subsidiar a los gobiernos Federal, Estatal y Municipal en la elaboración de políticas públicas en los diferentes sectores. Para ello se utilizará la base de datos en línea del Sistema de Tráfico Internacional (STI) del DPF La muestra estará conformada por datos quinquenales, desde el período del I de enero de 2008 al 3I de diciembre de 2012, a nivel nacional. Métodos de observación, entrevista informal, investigación bibliográfica, investigación documental y aplicación de cuestionarios. La recolección de datos primarios se realizó mediante acceso directo al STI. Las entrevistas se realizaron con is especialistas, funcionarios del ámbito federal, estatal y municipal, en las áreas de seguridad, educación y salud. Los especialistas señalaron categorías como útiles para el control del flujo migratorio, además de servir para cuantificar y dimensionar los servicios migratorios para adecuarse a los estándares internacionales, dado que se señaló como útil para el control del flujo migratorio, además de sirviendo para cuantificar y escalar los servicios de migración para adecuarlos a los estándares internacionales. Se concluyó que existe una falta de integración de las bases de datos de los sistemas informáticos públicos, lo que contribuye a un incremento en los costos de producción de información, debido a la dificultad de reutilizar los datos almacenados por otros órganos de la administración pública, siendo factible la práctica de aplicación de los datos respectivos actualmente disponibles.

PALABRAS CLAVE: Gestión pública. Tráfico internacional. Seguridad, salud; economía.

ABSTRACT: The objective of this work is to analyze the International Traffic System (STI) in order to identify the existing elements in the database subject to statistical treatment, able to subsidize the Federal, State and Municipal governments in the elaboration of public policies in the different sectors. For this, the online database of the DPF's International Traffic System (STI) will be used. The sample will consist of five-year data, from the period from January I, 2008 to December 31, 2012, at the national level. Observational methods, informal interview, bibliographic research, documentary research and questionnaire application. The collection of primary data was performed through direct access to the STI. The interviews were conducted with is specialists, civil servants from the federal, state and municipal spheres, in the areas of safety, education and health. The specialists pointed out categories as useful for the control of the migratory flow, in addition to serving to quantify and dimension the migration services to adapt to international standards, given that it was indicated as useful for the control of the migratory flow, in addition to serving to quantify and scale the services of migration to suit international standards. It was concluded that there is a

lack of integration of the databases of public IT systems, which contributes to an increase in the costs of producing information, due to the difficulty of reusing data stored by other public administration bodies, being feasible the application practice of the respective data currently available.

KEYWORDS: Public management. International traffic. Safety. Health. Economy.

\section{INTRODUÇÃO}

O Brasil é um país de dimensões continentais, constituído por 26 Estados e or Distrito Federal com 8.510.295,914 $\mathrm{km}^{2}$, sendo o quinto maior país do planeta e com $48 \%$ da área da América do Sul, contando com 5.568 municípios, mais o distrito insular de Fernando de Noronha. Dada essas dimensões e governos, é notório que se necessita da integração dos diversos órgãos setoriais para que se possa administrar os projetos e programas governamentais e gerar políticas públicas 
para o bem-estar social.

Os governos buscam a implementação de práticas de governança voltadas para a melhoria das condições sociais. Sob essa ótica, a elaboração de políticas públicas baseada em informações e indicadores se revela como um importante fator de contribuição para a eficácia e eficiência dos programas de governo e o efetivo direcionamento para a solução das demandas sociais.

No ano 2007, para o cumprimento de sua missão constitucional, foi implementado no Departamento de Polícia Federal (DPF) o novo Sistema do Tráfego Internacional (STI), passando a dispor de uma sólida, robusta, consistente e confiável base de dados capaz de proporcionar instrumentos para a elaboração de políticas públicas em diversos setores das três esferas de governo.

Uma base de dados de tráfego internacional, uma vez organizada e estatisticamente tratados de forma a possibilitar uma correta leitura e interpretação de seu conteúdo, servem de suporte para subsidiar a elaboração e políticas públicas aptas a melhorar as condições dos diversos segmentos da sociedade.

O Sistema do Tráfego Internacional (STI) realiza em tempo real e de forma "on-line" a coleta, atualização e armazenamento de dados referente à identificação pessoal do passageiro, classificação do viajante, objetivo da estada, tipo de documento de viagem, data de entrada e saída no território nacional, meio de transporte utilizado, identificação do transporte, histórico de viagens, origem e destino, dentre outras informações (Carvalho, 2015).

Atualmente, esses dados ficam restritos ao fornecimento de informações institucionais voltadas para as atividades fim do próprio DPF, não havendo o compartilhamento desses dados com outros setores públicos com vistas a elaboração de políticas públicas em benefício da sociedade.

O DPF possui uma preciosa fonte de informação capaz de ser utilizada de forma compartilhada pelos vários setores de governo, para fins de subsídio de elaboração de políticas públicas. Como, por exemplo, podem ser utilizados no cenário de pandemia.

Sob esta ótica, buscar-se-á no presente estudo, apresentar o novo STI utilizado pelo DPF, além de identificar e demonstrar que o mesmo dispõe de uma base de dados sólida, robusta, consistente e confiável.

Com relação ao turismo, o STI dispõe da possibilidade de realizar o tratamento estatístico de dados apresentando os períodos de alto e baixo fluxo de movimentação de entrada e saída de viajantes, classificados por objetivo de viagem (turismo, negócios, estudos, etc.), o que representa a possibilidade de elaboração de ações governamentais junto do trade turístico nacional, estadual e municipal, nos respectivos períodos.

Sob o aspecto da educação, o STI pode fornecer dados referentes a nacionalidades de viajantes que conjugado com a evolução do fluxo turístico, podem desencadear políticas públicas 
de promoção de cursos, a exemplo de idiomas, os quais podem ser projetados de acordo com a demanda da nacionalidade dos estrangeiros.

Com relação à saúde o tratamento de dados pode ser feito com vistas a identificar eventuais pontos de migração que concentram um maior fluxo de passageiros oriundos de determinada região do globo, que representem potenciais vetores de endemias, proporcionando aos órgãos de saúde das três esferas de governo a realização de barreiras sanitárias e eventual aquisição de insumos, medicamentos ou vacinas específicas.

No desempenho de sua missão constitucional relativa ao controle do tráfego migratório, o DPF realiza diariamente nos vários pontos de migração os registros eletrônicos da movimentação de entrada e saída de viajantes, especificando a sua classificação, objetivos da estada, estabelecendo prazos de permanência, além de praticar diversos outros atos administrativos inerentes às atividades de polícia judiciária e de polícia administrativa (Carvalho, 2015).

O processo de fiscalização e controle migratório segundo SERPRO (2011, p. 6) ocorre "através de 117 pontos de entrada, sendo alguns deles temporários, fiscalizados pela Polícia Federal, distribuídos pelo país, sendo 50 aeroportos internacionais, 42 portos marítimos e 25 postos terrestres".

A estrutura governamental na esfera do Poder Executivo Federal, em razão das tarefas atribuídas ao Departamento de Polícia Federal, avoluma-se em trabalho e reduz-se em resultados satisfatórios para as demandas sociais, uma vez que o órgão adota o denominado modelo burocrático de organização, com todos os aspectos positivos e negativos que a burocracia impõe aos administrados, conforme apresentado por CHIAVENATO (1999, p. 419), especialmente porque:

Segundo o conceito popular, a burocracia é visualizada geralmente como uma empresa ou uma organização onde o papelório se multiplica e se avoluma, impedindo as soluções rápidas ou eficientes. O termo também é empregado com o sentido de apego aos funcionários aos regulamentos e rotinas, causando ineficiência à organização. $\mathrm{O}$ leigo passou a dar o nome de burocracia aos defeitos do sistema (disfunções) e não ao sistema em si.

\section{Metodologia}

Trata-se de um estudo exploratório-descritivo com abordagem qualitativa e quantitativa, com o propósito de analisar o STI visando identificação de dados existentes que permitam subsidiar os órgãos públicos das três esferas de governo em desenvolver ações sociais a partir dos achados existentes, servindo como passo inicial, para a formação de uma nova perspectiva de trabalho mais moderna, ágil e segura, na produção e elaboração de políticas públicas mais eficientes e direcionadas a problemas específicos.

É uma pesquisa exploratória, pois procura aprimorar as propostas inicialmente formuladas 
para subsidiar pesquisas posteriores, além de buscar maiores informações acerca do problema em estudo.

Já o caráter descritivo, tem por objetivo primordial a descrição das características de determinada população ou fenômeno, e, em geral, utiliza técnicas estatísticas, e se baseia no fato de realizar a observação, registro e análise das possibilidades de utilização dos dados disponíveis.

A abordagem quantitativa é o método que gera um conjunto de dados que podem ser analisados através de técnicas matemáticas como é o caso das porcentagens. O método qualitativo é aquele no qual é importante a interpretação por parte do pesquisador com suas opiniões sobre o fenômeno em estudo (Pereira et al, 2018).

A base de dados "online" do Sistema do Tráfego Internacional (STI), do DPF. Esta base possui os dados relativos à: identificação pessoal do passageiro (data de nascimento, sexo, nacionalidade); classificação do viajante (turista, temporário, deportado, extraditado, tripulante, restos mortais, etc.); objetivo da estada (negócios, turismo, estudos, missão religiosa, etc), tipo de documento de viajem (passaporte, identidade, "laissez passer", etc.), data de entrada e saída no território nacional, meio de transporte utilizado, identificação do transporte, histórico de viagens, origem, destino, dentre outras informações (Carvalho, 2015).

Para Sá-Silva, Almeida e Guindani (2009):

[...] é fundamental que os (as) cientistas sociais entendam o significado de fontes primárias e fontes secundárias. As fontes primárias são dados originais, a partir dos quais se tem uma relação direta com os fatos a serem analisados, ou seja, é o pesquisador (a) que analisa. Por fontes secundárias compreende-se a pesquisa de dados de segunda mão, ou seja, informaç̃̃es que foram trabalhadas por outros estudiosos e, por isso, já são de domínio científico, o chamado estado da arte do conhecimento.

\section{I Procedimentos de coleta de dados}

Envolveu as seguintes etapas:

I. Obtenção de permissão para acesso à base de dados e assinatura do fiel depositário.

2. A realização de pesquisa documental, com a identificação da legislação aplicável, envolvendo Leis Federais, projetos de lei, resoluções e outros atos normativos em geral, bem como, outros documentos oficiais referentes à implementação de políticas públicas que guardem pertinência com o assunto sob estudo, buscando identificar novos materiais que ainda não tiveram tratamento adequado a matéria.

3. Utilização de dados primários. A Coleta de dados será realizada mediante acesso direto ao STI pelo próprio pesquisador para a extração dos dados necessários ao cumprimento dos objetivos apresentados para o presente estudo.

4. Realização de entrevista com is especialistas que trabalham nas esferas dos governos: 
federal, estadual e municipal. Destes, 07 são da área da segurança, 04 da área da saúde e 04 da área da educação.

\section{Elaboração do relatório final.}

Tabela I - Entrevistados.

\begin{tabular}{|l|l|l|}
\hline Esfera do governo & Número de entrevistados & $\begin{array}{l}\text { Tempo de experiência } \\
\text { (média em anos) }\end{array}$ \\
\hline Federal & 8 & 20 anos \\
\hline Estadual & 6 & II anos \\
\hline Municipal & I & Io anos \\
\hline
\end{tabular}

Fonte: elaboração própria.

\section{Resultados}

Os quinze especialistas, das três áreas, opinaram sobre quais informações e dados seriam importantes para tornar o STI mais completo e eficaz para tomada de decisões, dentre os dados existentes, e opinando quais dados novos poderiam completar o STI, veja Tabela 3.

$\mathrm{Na}$ Tabela 2, eles analisaram os dados já existentes, quais seriam importantes, respondendo SIM, conforme suas áreas de atuação para tomada de decisões.

Tabela 2 - Categoria de dados existentes no STI

\begin{tabular}{|l|l|l|l|}
\hline $\begin{array}{l}\text { CATEGORIA DE DADOS } \\
\text { EXISTENTES }\end{array}$ & SAÚDE & SEGURANÇA & EDUCAÇÃO \\
\hline Tipo de Movimento & SIM & SIM & SIM \\
\hline Tipo de Fronteira & SIM & SIM & NÃO \\
\hline Data do Atendimento & SIM & SIM & SIM \\
\hline Tipo do Transporte & SIM & SIM & NÃO \\
\hline Data do Transporte & SIM & SIM & NÃO \\
\hline Identificador do Transporte & SIM & SIM & NÃO \\
\hline
\end{tabular}




\begin{tabular}{|c|c|c|c|}
\hline Tipo do Documento & NÃO & SIM & $\mathrm{NA \tilde {O }}$ \\
\hline Número do Documento & SIM & SIM & NÃO \\
\hline Data de Validade & NÃO & SIM & $\mathrm{NÃO}$ \\
\hline RNE & NÃO & SIM & NÃO \\
\hline Validade da CIE & NÃO & SIM & $\mathrm{NÃO}$ \\
\hline Nome & SIM & SIM & $\mathrm{NA} \mathrm{O}$ \\
\hline Data de Nascimento & SIM & SIM & SIM \\
\hline Sexo & SIM & SIM & $\mathrm{NÃO}$ \\
\hline País de Nacionalidade & SIM & SIM & SIM \\
\hline Classificação & SIM & SIM & SIM \\
\hline Prazo de Estada & SIM & SIM & NÃO \\
\hline Prazo de Ausência & NÃO & SIM & NÃO \\
\hline
\end{tabular}

Fonte: elaboração própria, categorias extraídas do STI.

As novas categorias de dados sugeridas pelos especialistas das áreas da saúde, educação e segurança pública foram: profissão, endereço de estada, declaração de valores, bagagem, acompanhante(s) da viagem, tempo médio de permanência no Brasil, tempo médio de ausência do Brasil, sistema de imunização, religião, outras línguas estrangeiras/idiomas, seguro saúde, doença grave, tempo de viagem, país de origem, raça/cor e grau de instrução.

Tabela 3 - Categoria de dados necessários ao STI

\begin{tabular}{|l|l|l|l|}
\hline CATEGORIA DE DADOS NECESSÁRIOS & SAÚDE & SEGURANÇA & EDUCAÇÃO \\
\hline Bagagem (Peso/Volumes) & SIM & SIM & SIM \\
\hline
\end{tabular}




\begin{tabular}{|c|c|c|c|}
\hline Endereço de estada & SIM & SIM & SIM \\
\hline Declaração de valores & SIM & SIM & SIM \\
\hline Acompanhantes de Viagem & SIM & SIM & SIM \\
\hline Tempo Médio de PERMANÊNCIA no Brasil & SIM & SIM & SIM \\
\hline Tempo Médio de ausência no Brasil & NÃO & SIM & SIM \\
\hline Profissão/Ocupação & SIM & SIM & SIM \\
\hline Sistema de Imunização & SIM & SIM & SIM \\
\hline Religião & SIM & NÃO & SIM \\
\hline Outras Línguas Estrangeiras/Idiomas & SIM & SIM & SIM \\
\hline Seguro Saúde & SIM & NÃO & SIM \\
\hline Doença grave & SIM & SIM & SIM \\
\hline Tempo de viagem & SIM & SIM & SIM \\
\hline
\end{tabular}




\begin{tabular}{|l|l|l|l|}
\hline Pais de Origem & SIM & SIM & SIM \\
\hline Raça/Cor & SIM & NÃO & NÃO \\
\hline Grau de Instrução & SIM & SIM & SIM \\
\hline
\end{tabular}

Fonte: Elaborado pelo autor.

De acordo com os dados apresentados na Tabela 3, verifica-se que a quase totalidade (I4 dos I5 entrevistados) atua diretamente na área de gestão pública ou privada, o que tende a ser um bom indicativo para a pesquisa, haja vista disponibilizar aos participantes uma visão ampla das atividades desenvolvidas por suas instituições.

A pesquisa mostrou que houve convergência nas três áreas para os dados: tipo de movimento, data do atendimento, data de nascimento, país de nacionalidade e classificação do viajante, sendo que nas demais categorias de dados a concordância foi parcial.

Para o dado "tipo de movimento", que representa a entrada ou a saída de um viajante no território nacional, os profissionais da área da saúde atribuíram o conceito "muito importante" para o controle do tráfego internacional, em razão de potenciais riscos sanitários ao país. Também foi apresentada a justificativa de que os dados de entrada contribuem para a realização de estudos e investigação de doenças ou epidemias.

Já para os profissionais da área de segurança pública, o dado relacionado ao "tipo de movimento" se justifica no setor operacional, com objetivo de se obter a movimentação em território nacional ou a saída de pessoas objeto de investigação.

No plano político-administrativo, o dado foi apontado como útil para o controle do fluxo migratório, além de servir para quantificar e dimensionar os serviços de migração para adequação dos padrões internacionais de conforto nas instalações dos pontos físicos de atendimento e tempo de despacho migratório.

A informação sobre o "tipo de fronteira" - que pode ser classificada em razão da via utilizada para o processo migratório, podendo ser utilizadas as vias: terrestre, marítima, aérea, fluvial ou lacustre - foi destacada pelos entrevistados da área da saúde como importante para o controle de ingresso de doenças no país, servindo como suporte para a indicação dos locais mais aptos ao desenvolvimento de campanhas de prevenção e orientação dos viajantes, bem como para a realização de ações de combate ao ingresso de potenciais vetores de doenças típicas de outros países. 
Já para o setor de segurança pública, a importância do dado se dá pela contribuição ao monitoramento dos tipos de fronteiras que possuem maior fluxo de migrantes.

A "data de atendimento" foi destacada como muito importante para a área da saúde, visto que, a origem ou o local de partida do viajante permite associar eventuais doenças transmissíveis com o período de incubação e picos de transmissão da doença.

Para o setor da segurança, esse dado permite planejar a melhor forma de dimensionamento $e$ movimentação dos efetivos de pessoal considerados escassos.

Para os especialistas da educação, os dados acima, são considerados relevantes, mas no sentido de se ter quantificação de migrantes.

Com relação ao conjunto de dados do transporte caracterizados por: tipo (ônibus, carro, navio, barco, avião, helicóptero e outros); data; e, identificador (placa do veículo, matrícula da embarcação, prefixo da aeronave, número do voo, entre outros) importantes observações foram apresentadas pelos profissionais da área da saúde, dentre as quais a informação de que o longo tempo de percurso de uma viagem pode acarretar carência de vitaminas e baixar a imunidade do indivíduo.

Os profissionais da área de segurança apontam como razões de importância os conjuntos de dados do transporte, como sendo úteis para à identificação de pessoas ou meios de transporte sob investigação que podem servir como fator para identificação de rotas de trânsito e vias de fuga utilizados por criminosos ou por pessoas a estes associados.

Não foram apresentadas contribuições relevantes sobre os dados do transporte pelos profissionais da área da educação.

Com relação aos dados do viajante, destaca-se que a parte relativa à documentação pessoal do passageiro, como o tipo, número e data de validade do documento de viagem, bem como o número do registro do estrangeiro (RNE) e respectiva data de validade, foram considerados como itens de pouca importância para os profissionais das áreas educação e saúde, sendo destacado por esses últimos profissionais como relevante apenas no sentido de servir para uma possível identificação correta de eventual vetor de doença infectocontagiosa.

Os mesmos dados foram considerados importantes para os entrevistados da área de segurança pública, uma vez que, além de auxiliar na correta identificação e individualização do passageiro, ajudam no processo de qualificação civil do indivíduo, possibilitando a realização dos trabalhos de prevenção na entrada de criminosos no país.

Os profissionais da segurança ligados ao DPF destacaram ainda a importância dos dados documentais do viajante, em especial os relativos aos estrangeiros, para o acompanhamento de eventuais pendências de natureza administrativa ou judiciais, dado que auxiliam por ser um argumento facilitador no processo de busca gerando uma maior precisão na realização dos 
procedimentos administrativos.

Dentre os dados dos viajantes os que mais se destacaram como de grande importância e extrema relevância para às três áreas (saúde, educação e segurança pública) destacam-se os dados qualificativos identificados pelos campos: nome, data de nascimento, sexo, país de nacionalidade.

Quanto à data de nascimento, os entrevistados da área de saúde entendem ser um dado primordial para identificar a idade dos viajantes.

Os profissionais da área educacional entendem que a data de nascimento em conjunto com outros dados citados, como o sexo, nacionalidade e motivo da viagem, podem servir para se traçar um perfil do viajante possibilitando o desenvolvimento de campanhas educativas com foco na idade além de possibilitar a identificação de outros motivos para a entrada ou saída do indivíduo no país.

A pesquisa revelou que a identificação do gênero se faz necessária para pesquisas na área de saúde, em razão da possibilidade de se estabelecer a relação deste dado com outras variáveis, resultando em informações específicas. Já para os profissionais da área da educação foi apontado como a possibilidade de identificar o perfil do turista, tendo como referência o sexo para possíveis campanhas de combate à exploração sexual.

A identificação do país de nacionalidade do viajante foi considerada pelos entrevistados da área da saúde como sendo um dado necessário para se identificar os riscos à saúde pública, trazidos pelos viajantes estrangeiros e que podem ser levados do Brasil para o exterior.

Os entrevistados do setor de segurança pública entendem que a identificação da nacionalidade dos indivíduos que migram no território nacional possui um relevante valor para o controle do fluxo migratório e adoção de medidas de segurança pública e de defesa.

Os especialistas em educação entendem que a nacionalidade pode subsidiar o planejamento na oferta de cursos de capacitação em idiomas ou, ainda, servir para redimensionar o número das vagas existentes, principalmente no setor privado que atua na capacitação de línguas estrangeiras.

Os profissionais da área da segurança pública indicam que a classificação do viajante funciona como mais um dado importante para fins de correta identificação do passageiro, os entrevistados da área da educação defendem que esta pode ser útil para a realização de estudos com objetivo de promover adaptações linguísticas de acordo com a nacionalidade para, principalmente, os residentes no Brasil.

Quanto aos dados do movimento relativo ao prazo de estada, os profissionais da saúde entendem que se trata de um dado útil em razão de poder indicar o tempo de exposição a fatores de risco ao qual estaria sujeito o viajante, também podendo ter utilidade para realizar investigação sobre determinado tipo de vírus ou bactéria que eventualmente ingressou no país, incubado em passageiro infectado, além de poder-se detectar o período de ingresso e a origem, mediante o confronto com 
outros dados como o local de entrada e o tipo de transporte.

Os profissionais da área de segurança pública, em especial os que atual diretamente com as atividades de migração, esclarecem que o dado possui relevância para demonstrar a dinâmica da movimentação do estrangeiro e analisar a compatibilidade do período de estada concedido ou solicitado com o tipo de visto apresentado, ou motivo de viagem declarado.

Com relação ao prazo de ausência, os profissionais da área de saúde destacam que o viajante que sai do Brasil passa a ser uma preocupação para a vigilância sanitária do país de destino.

\section{Conclusão}

As modificações na sistemática de uso do STI são necessárias para que as informações possam ser utilizadas pelos três níveis de governo, isso devido ao fato de que na sociedade contemporânea busca a eficiência das ações do governo.

Os dados servem de suporte para subsidiar a elaboração de políticas governamentais, possibilitando a melhoria das condições em diversos segmentos da sociedade, fazendo com que o governo em sua tríplice esfera possa atender às necessidades sociais de forma rápida e segura, oferecendo maiores benefícios para a sociedade, com a redução de riscos e custos.

Os dados do STI estão aptos a subsidiar os governos Federal, Estadual e Municipal na elaboração de políticas públicas nos diversos setores, inclusive na área da saúde. Para o Departamento de Polícia Federal possibilita a construção, em tempo real, de diversos cenários, utilizando os dados do STI.

Por meio dessas informações contendo os dados pessoais e a classificação do viajante em razão do visto apresentado, permite-se concluir que existem diversas possibilidades de desenvolvimento de estudos envolvendo o perfil do viajante sobre vários aspectos, como, por exemplo: gênero, idade, trabalho, estudo, fixação de residência, duração de viagem, os reflexos na economia local, períodos de maior e menor fluxo migratório, evolução de demandas locais, adensamentos populacionais, incidência de viajante por nacionalidade, identificação do fluxo de passageiros nos pontos de entrada/saída.

Há uma necessidade de maior integração das bases de dados do STI com outros sistemas públicos mantidos pelo governo federal, a exemplo do Sistema Nacional de Registro de Hóspedes e do Sistema de Emissão de Vistos Consulares, de forma a ampliar o potencial de uso do STI com o consequente aumento do valor das informações por ele geradas.

As novas categorias de dados apresentadas pelos especialistas entrevistados, nas três áreas para os dados: profissão, endereço de estada, declaração de valores, bagagem, acompanhantes da viagem, tempo médio de permanência no Brasil, sistema de imunização, outras línguas, estrangeiras/idiomas, doença grave, tempo de viagem, país de origem e grau de instrução. 
A limitação apresentada neste trabalho servirá de incentivo a outros pesquisadores que podem se utilizar do modelo aqui seguido para a realização de novos estudos similares que venham ampliar as respostas, bem como fortalecer as informações obtidas na atual investigação.

Sugere-se se a administração do DPF na formulação de convênios específicos com os interessados no acesso aos dados, possibilite a disponibilização por plataforma eletrônica mediante processo de autenticação e controle do pesquisador ou do órgão interessado em obter os dados quantitativos.

\section{REFERÊNCIAS}

BASTOS, Núbia M. Garcia. Introdução à metodologia do trabalho acadêmico. $4 \cdot^{\underline{a}}$ ed. Gráfica e Editora Nacional: Fortaleza, 2007.

BRAGA, Pedro. Ética, direito e administração pública. Brasília: Senado Federal, Subsecretaria de edições técnicas, 2006.

BRASIL. Constituição (1988). Constituição da República Federativa do Brasil. Brasília, DF, Senado, 2010. . SERPRO. Manual do Usuário STI - Versao 6.I. [s.ed.], 201 I.

CARVAlHO, P. R. L. Sistema do Tráfego Internacional: Subsídio para as Políticas Públicas nos Multisetores da Tríplice Esfera Governamental. 2015, I44 fls. Dissertação de Mestrado Profissional. Universidade Estadual do Ceará, Centro de Estudos Sociais Aplicados. Fortaleza, 2015.

CASA CIVIL. Medidas adotadas pelo Governo Federal no combate ao coronavírus - 3I de março. Publicado em or/o4/2020; Atualizado em 28/04/2020. Medidas adotadas pelo Governo Federal no combate ao coronavírus - 3I de março - Português (Brasil) (www.gov.br). Acesso em: 15.02.202I.

CHIAVENATO, Idalberto. Introdução à teoria geral da administração. $5^{\underline{a}}$ ed. Campus editora: Rio de Janeiro, 1999.

PERERIA, A.S. et al. (2018). Metodologia da pesquisa científica. [e-book]. Santa Maria. Ed. $\mathrm{UAB} / \mathrm{NTE} / \mathrm{UFSM}$.

SILVA. Régis de Albuquerque; ELOY DA COSTA, Odorico de Moraes. A modernização da Gestão Pública no Ceará: as contribuições do Programa Estadual de Incentivo às Organizações Sociais. Conhecer: debate entre $o$ público $e \quad o$ privado. V.I, n. ${ }^{\circ}$, 2011. $\langle$ http://www.seer.uece.br/?journal=politicaspublicasemdebate\&page $=$ article\&op=view \& path[] 63 $>$. Acesso em ir jan. 2013. 